\title{
What's in a Relationship: An Ontological Analysis
}

\author{
Giancarlo Guizzardi ${ }^{1}$, Gerd Wagner ${ }^{2}$ \\ ${ }^{1}$ Comp. Science Department, Federal University of Espírito Santo (UFES), Brazil \\ ${ }^{2}$ Brandenburg University of Technology at Cottbus, Germany \\ gguizzardi@inf.ufes.br,wagnerg@tu-cottbus.de
}

\begin{abstract}
In a series of publications, we have proposed a foundational system of ontological categories which has been successfully used to evaluate and improve the quality of conceptual modeling grammars and models. In this article, we continue this work by using this foundational ontology to provide real-world semantics and sound modeling guidelines for one of the most fundamental (and yet one of the most problematic) constructs in conceptual modeling, namely, the relationship type. In addition, we systematically compare our approach with a classical ontological treatment of this construct in the literature, provided by the BWW framework.
\end{abstract}

\section{Introduction}

In recent years, there has been a growing interest in the application of Foundational Ontologies, i.e., formal ontological theories in the philosophical sense, for providing real-world semantics for conceptual modeling languages, and theoretically sound foundations and methodological guidelines for evaluating and improving the individual models produced using these languages.

For a number of years, we have been developing a foundational ontology named UFO (Unified Foundational Ontology) [1-3] by employing theories from Formal Ontology, Cognitive Psychology, Linguistics, Philosophy of Language and Philosophical Logics. In a series of publications, this reference ontology has been successfully applied to analyze a number of fundamental conceptual modeling constructs ranging from Roles, Types and Taxonomic Structures, Part-Whole Relations, Attributes, Weak Entities and Datatypes, among others. The system of ontological categories constituting UFO is presented in depth in [1], together with its empirical justifications and formal characterization.

In this article we continue this work by addressing one of the most fundamental (and yet one of the most problematic) constructs in conceptual modeling, namely, the relationship type (also named "association" or "relation"). Despite its importance, empirical evidence shows that the use of this construct is often problematical as a way of communicating meaning in an application domain [4]. In pace with [5], we believe that this is mainly due to the lack of consensus and imprecise definitions of its realworld semantics.

The remaining of this article is organized as follows. In section 2, we present the core categories of the UFO ontology, focusing on those aspects which are germane to the purpose of this article. In particular, in sections 2.4 and 2.5 we built on the work 
presented in [2] to propose an ontological theory of relations. In section 3, we employ the theory presented in section 2 to provide an ontological analysis of relationship types and well-founded guidelines for their representation in conceptual models. In section 4 , we briefly compare the results of section 3 with a classical ontological treatment of this construct in the literature, provided by BWW framework. Section 5 presents some final considerations.

\section{Background: The Unified Foundational Ontology (UFO)}

The core of the UFO ontology is depicted in figure 1 below. A fundamental distinction in this ontology is between the categories of Individual and Universal. Individuals are entities that exist in reality possessing a unique identity. Universals, conversely, are space-time independent pattern of features, which can be realized in a number of different individuals. The core of this ontology exemplifies the so-called Aristotelian ontological square comprising the category pairs Object-Object Universal, Trope-Trope Universal. From a metaphysical point of view, this choice allows for the construction of a parsimonious ontology, based on the primitive and formally defined notion of existential dependence [1]: Definition 1 (existential dependence): Let the predicate $\varepsilon$ denote existence. We have that an individual $x$ is existentially dependent on another individual $y$ (symbolized as $e d(x, y)$ ) iff, as a matter of necessity, $y$ must exist whenever $x$ exists, or formally (1). ed(x,y) $=_{\text {def }} \square(\boldsymbol{\varepsilon}(\mathbf{x}) \rightarrow \boldsymbol{\varepsilon}(\mathbf{y}))$. In complementary manner, we define two individuals as independent from each other as: (2). $\operatorname{indep}(x, y)=_{\text {def }} \neg \operatorname{ed}(x, y) \wedge \neg \operatorname{ed}(y, x)$.

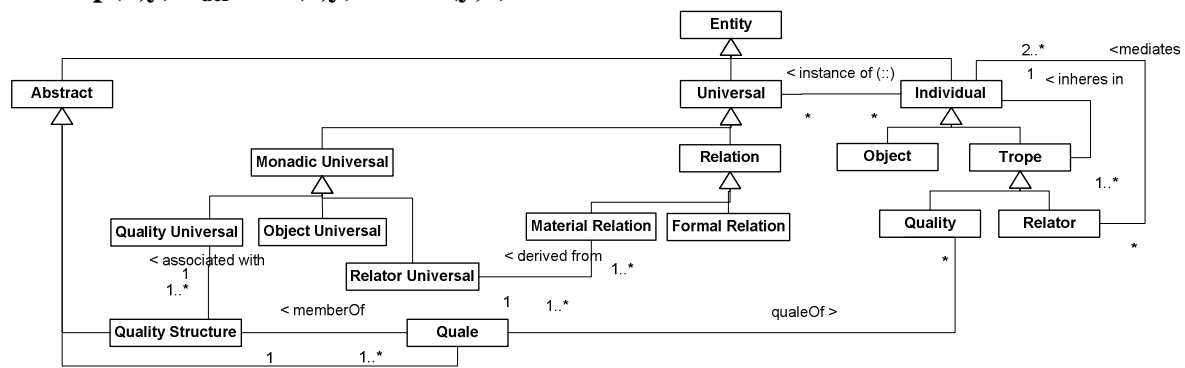

Figure 1. Excerpt of the Foundational ontology UFO.

\subsection{Tropes and Objects}

Intuitively, a trope is an instance of a property (i.e., the instance of an objectified property) of a specific entity: the redness of John's T-shirt is a trope that inheres to John's T-shirt (the host). Both John's T-shirt and the redness of John's T-shirt are individuals. However, they are individuals of very different natures. Tropes are individuals which can only exist in other individuals, i.e., they are existentially dependent on other individuals in the way, for instance, the color and the weight of an apple $a$ depend on $a$, the electric charge of a conductor $c$ depends on $c$, or John's headache depends on John. In contrast, individuals such as John, the apple $a$, and the conductor 
$c$ do not inhere in other individuals and, hence, are not existentially dependent entities in this sense. In this article, we give the name Object to the latter type of individual.

As discussed in [1], there is solid evidence for tropes in the literature. On one hand, in the analysis of the content of perception, tropes are the immediate objects of everyday perception. On the other hand, the idea of tropes as truthmakers underlies a standard event-based approach to natural language semantics. The notion of tropes employed here comprises: (a) Intrinsic Tropes or Qualities: an individualized (objectified) color, temperature, or weight, a symptom, a skill, a belief, an intention, an electric charge; (b) Relational Tropes or Relators: a kiss, a handshake, a covalent bond, a medical treatment, but also social objects such as an enrollment, an employment, a purchase order and a commitment or claim.

Existential dependence can also be used to differentiate intrinsic and relational tropes: qualities are dependent on one single individual; relators depend on a plurality of individuals. More technically, a special type of existential dependence relation that holds between a trope $x$ and the individual $y$ of which $x$ depends is the relation of inherence $(i)$. Thus, for an individual $x$ to be a trope of another individual $y$, the relation $i(x, y)$ must hold between the two. For example, inherence glues your smile to your face, or the charge in a specific conductor to the conductor itself. We then formally characterize a trope as an individual that inheres in (and, hence, is existentially dependent upon) another individual: Definition 2 (Trope): (3). Trope(x) $=_{\text {def }}$ Individual $(\mathbf{x}) \wedge \exists \mathbf{y} i(\mathbf{x}, \mathbf{y})$.

Inherence is irreflexive, asymmetric and intransitive relation. Moreover, in our framework, we adopt the so-called non-migration (or non-transferability) principle. This means that it is not possible for a trope $m$ to inhere in two different individuals $a$ and $b$ : (4). $\forall \mathbf{x}, \mathbf{y}, \mathbf{z}$ (Trope(x) $\wedge i(\mathbf{x}, \mathbf{y}) \wedge i(\mathbf{x}, \mathbf{z}) \rightarrow \mathbf{y}=\mathbf{z})$. The unique individual $y$ that a tropes $x$ inheres in is termed the bearer of $x$ and is defined as follows: Definition 3 (Bearer of a Trope) ${ }^{1}:(\mathbf{5}) . \boldsymbol{\beta}(\mathbf{x})==_{\operatorname{def}}$ ty $\boldsymbol{i}(\mathbf{x}, \mathbf{y})$. The bearer of a trope can itself be another trope. Examples include the individualized time extension, or the gravity of John's headache. The infinite regress in the inherence chain is prevented by the fact that there are individuals (namely Objects) that cannot inhere in other individuals.

\subsection{Qualia and Quality Structures}

The feature of tropes defined by the non-migration principle (formula 4) seems at first counterintuitive. For example, if we have two particulars $a$ (a red apple) and $b$ (a red car), and two tropes $\mathrm{r}_{1}$ (particular redness of $a$ ) and $\mathrm{r}_{2}$ (particular redness of $b$ ), we consider $r_{1}$ and $r_{2}$ to be different individuals, although perhaps qualitatively indistinguishable. What does it mean then to say that $a$ and $b$ have the same color? Due to (4), sameness here cannot refer to strict (numerical) identity, but only to a qualitative one (i.e., equivalence in a certain respect). We thus distinguish between the color of a particular apple (its quality) and its 'value' (e.g., a particular shade of red). The latter is named quale, and describes a projection of an individual quality into a certain value space or measurement structure named a quality structure [1].

\footnotetext{
The iota operator ( 1 ) used in a formula such as $\mathrm{x} \varphi \varphi$ was defined by Bertrand Russel and implies both the existence and the uniqueness of an individual $x$ satisfying predicate $\varphi$.
} 
An attempt to model the relation between properties and their representation in human cognitive structures is presented in the theory of conceptual spaces introduced in [6]. The idea is that for several perceivable or conceivable quality universals there is an associated quality structure in human cognition. For example, height and mass are associated with one-dimensional structures with a zero point isomorphic to the half-line of nonnegative real numbers. Other properties such as color and taste are represented by multi-dimensional structures. Moreover, [6] defends that this notion should be understood literally, i.e., quality structures are endowed with certain geometrical properties (topological or ordering structures) that constrain the relations between its constituting dimensions. For example, both the dimensions of height and mass are totally ordered structures. For an in depth discussion on the topic of quality structures and their role in conceptual modeling one should refer to $[1,2]$.

\subsection{Relations and Relators}

Relations are entities that glue together other entities. Every relation has a number of relata as arguments, which are connected or related by it. The number of a relation's arguments is called its arity. In the philosophical literature, two broad categories of relations are typically considered, namely, material and formal relations $[7,8]$.

Formal relations hold between two or more entities directly, without any further intervening individual. In principle, it includes those relations that form the mathematical superstructure of our framework. Examples include existential dependence $(e d)$, inherence $(i)$, subtype-of, part-of, subset-of, instantiation(::), among many others not discussed here [1]. We name these relations here basic formal relations [7]. However, we also classify as formal those domain relations that exhibit similar characteristics, i.e., those relations of comparison such as is taller than, is older than, knows more Greek than. We name these relations domain formal relations. As pointed out in [8], the entities that are immediate relata of such relations are not objects but qualities. For instance, the relation heavier-than between two atoms is a formal relation that holds directly as soon as the relata (atoms) are given. The truth-value of a predicate representing this relation depends solely on the atomic number (a quality) of each atom and the material content of heavier-than is as it were distributed between the two relata. Moreover, to quote [8], "once the distribution has been effected, the two relata are seen to fall apart, in such a way that they no longer have anything specifically to do with each other but can serve equally as terms in a potentially infinite number of comparisons".

Material relations, conversely, have material structure on their own and include examples such as working at, being enrolled at, and being connected to. Whilst a formal relation such as the one between Paul and his knowledge $x$ of Greek holds directly and as soon as Paul and $x$ exist, for a material relation of being treated in between Paul and the medical unit $\mathrm{MU}_{1}$ to exist, another entity must exist which mediates Paul and $\mathrm{MU}_{1}$. We name these entities relators. Relators are individuals with the power of connecting entities. For example, a medical treatment connects a patient with a medical unit; an enrollment connects a student with an educational institution; a covalent bond connects two atoms. The notion of relator (relational tropes) is supported by several works in the philosophical literature $[7,8]$ and, the position advocated here is that they play an important role in answering questions of the sort: what 
does it mean to say that John is married to Mary? Why is it true to say that Bill works for Company X but not for Company Y?

An important notion for the characterization of relators (and, hence, for the characterization of material relations) is the notion of foundation. Foundation can be seen as a type of historical dependence [1], in the way that, for instance, an instance of being kissed is founded on an individual kiss, or an instance of being punched by is founded on an individual punch, an instance of being connected to between airports is founded on a particular flight connection.

Suppose that John is married to Mary. In this case, we can assume that there is an individual relator (relational trope) $\mathrm{m}_{1}$ of type marriage that mediates John and Mary. The foundation of this relator can be, for instance, a wedding event or the signing of a social contract between the involved parties. In other words, for instance, a certain event $\mathrm{e}_{1}$ in which John and Mary participate can create an individual marriage $\mathrm{m}_{1}$ which existentially depends on John and Mary and which mediates them. The event $\mathrm{e}_{1}$ in this case is the foundation of relator $\mathrm{m}_{1}$.

Now, let us elaborate on the nature of the relator $m_{1}$. There are many qualities that John acquires by virtue of being married to Mary. For example, imagine all the legal responsibilities and rights that John has in the context of this relation. These newly acquired tropes are intrinsic qualities of John which, therefore, inhere and are existentially dependent on him. However, these qualities also depend on the existence of Mary. We name this type of qualities externally dependent qualities, i.e., externally dependent qualities are intrinsic tropes that inhere in a single individual but that are existentially dependent on (possibly a multitude of) other individuals: Definition 4 (Externally Dependent Quality): A quality $x$ is externally dependent iff it is existentially dependent of an individual which is independent of its bearer. Fornally, (6).

$\operatorname{ExtDepQuality}(\mathbf{x})=_{\operatorname{def}} \operatorname{Quality}(\mathbf{x}) \wedge \exists \mathbf{y} \operatorname{indep}(\mathbf{y}, \boldsymbol{\beta}(\mathbf{x})) \wedge \operatorname{ed}(\mathbf{x}, \mathbf{y})$.

In the same manner, there are also a number of individual qualities (e.g., rights and responsabilities) that Mary acquires by virtue of being married to John. Now, we can define an aggregate $\mathrm{m}_{1}$ composed of all these externally dependent qualities that share the same foundation. In this example, $\mathrm{m}_{1}$ is exactly the sum of all qualities (rights and responsabilities) acquired by John and Mary due to the same foundational event, i.e., $\mathrm{m}_{1}$ is the instance of the relational property marriage that mediates John and Mary and that is the truthmaker of propositions such as "John is married to Mary", "Mary is married to John", "John is the husband of Mary", and "Mary is the wife of John".

A relator is said to mediate (or connect) the relata of a material relation (symbolized by $m(x, y))$. As discussed above, mediation is a special type of existential dependence relation or, more specifically, a sort of non-exclusive inherence (see [1] for formal details). Finally, we require that a relator mediates at least two distinct individuals, i.e., (7). $\forall \mathbf{x} \operatorname{Relator}(\mathbf{x}) \rightarrow \exists y, w(y \neq w \wedge m(x, y) \wedge m(x, w))$.

\subsection{Universals}

An Object Universal is a universal whose instances are objects (e.g., the universal Person or the universal Apple). A Quality Universal is a universal whose instances are individual qualities (e.g., the objectified color of this apple is an instance of the universal color, a particular headache is an instance of the universal Symptom), and a Relator Universal is one whose instances are individual relational tropes (e.g., the 
particular enrollment connecting John and a certain University is an instance of the universal Enrollment). Finally, a Relation is a universal whose instances are n-tuples or related elements (e.g., being older than, being married to, being the father of).

In general, conceptual specifications (such as UML class diagrams and ER specifications) represent conceptualizations only at the type level, i.e., only universals and relations among universals are typically represented. Thus, we define the formal relations of Characterization and Mediation as the counterparts at the type level of the relations inheres in and mediates, respectively. In these definitions, the symbol :: represents the formal relation of instantiation: Definition 5 (Characterization): A universal $\mathrm{U}$ is characterized by a trope universal $\mathrm{T}$ iff every instance of $\mathrm{U}$ bears an instance of T. Formally, (8). charac $(\mathbf{U}, \mathbf{T})=_{\text {def }} \operatorname{Universal}(\mathbf{U}) \wedge$ QualityUniversal $(\mathbf{T})$ $\wedge \forall \mathbf{x}(\mathbf{x}:: \mathrm{U} \rightarrow \exists \mathbf{y} \mathbf{y}:: T \wedge i(\mathbf{y}, \mathbf{x}))$; Definition 6 (Mediation): The mediation relation holds between a universal $U$ and a relator universal $U_{R}$ iff every instance of $U$ is $m e$ diated by $(\mathrm{m})$ an instance of $\mathbf{U}_{\mathbf{R}}$. Formally, (9). mediation $\left(\mathbf{U}, \mathbf{U}_{\mathbf{R}}\right)=_{\text {def }} \operatorname{Universal}(\mathbf{U}) \wedge$ RelatorUniversal $\left(U_{R}\right) \wedge \forall \mathbf{x}\left(\mathbf{x}:: U \rightarrow \exists r\right.$ r:: $\left.U_{R} \wedge m(r, x)\right)$.

Relator universals constitute the basis for defining material relations $\mathrm{R}$ whose instances are n-tuples of entities. In general, a material relation $\mathrm{R}$ can be defined by the following schema: Definition 7 (Material and Formal Relations): Let $\phi\left(a_{1}, \ldots, a_{n}\right)$ denote a condition on the individuals $\mathrm{a}_{1}, \ldots, \mathrm{a}_{\mathrm{n}}$

$$
\left[a_{1} \ldots a_{n}\right]:: R\left(U_{1} \ldots U_{n}\right) \leftrightarrow \wedge_{i \leq n} a_{i}:: U_{i} \wedge \phi\left(a_{1} \ldots a_{n}\right)
$$

A relation is called material if there is a relator universal $U_{R}$ such that the condition $\phi$ is obtained from $U_{R}$ as follows: $\left.\phi\left(\mathbf{a}_{1} \ldots \mathbf{a}_{\mathbf{n}}\right) \leftrightarrow \exists \mathbf{k}\left(\mathbf{k}:: U_{R} \wedge_{\mathbf{i}} \leq \mathbf{n} \mathbf{m}\left(\mathbf{k}, \mathbf{a}_{\mathbf{i}}\right)\right)\right)$. In this case, we say that the relation $R$ is derived from the relator universal $U_{R}$, or symbolically, derivation $\left(R, U_{R}\right)$. Otherwise, if such a relator universal $U_{\mathrm{R}}$ does not exists, $\mathrm{R}$ is termed a formal relation.

We can summarize this discussion as follows: (1) we make a fundamental distinction between formal and material relations. Whilst the former hold directly between two entities without any further intervening individual, the latter are induced by mediating entities called relators. Moreover, material relations are founded by material entities in reality, typically events, which are external to their relata. Domain formal relations, in contrast, are founded in qualities which are intrinsic to the their relata and, hence, can be reduced to relations between these qualities; (2) Relators are special types of (relational) tropes, i.e., particularized relational properties and are aggregations of externally dependent qualities; (3) Externally dependent qualities exemplify the properties that an individual has in the scope of a certain material relation; (4) We explicitly differentiate a relator universal from the material relations (classes of tuples) derived from that relator universal.

\section{An Ontological Foundation for Conceptual Modeling Relations}

In this section, we employ the set of ontological categories proposed is section 2 to analyze and provide foundations for conceptual modeling relationship types or relations. These modeling concepts are represented in practically all conceptual modeling languages. Thus, the conclusions drawn in what follows can be extended to all these 
languages. However, with the sole purpose of exemplification, we shall refer in the sequel to these concepts as they are represented by UML's modeling primitives.

In most conceptual modeling languages, $n$-ary relationship types are taken to represent sets of n-tuples. In UML, the ER concept of a relationship type is called association: "an association defines a semantic relationship that can occur between typed instances...An instance of an association is called a link...An association declares that there can be links between instances of the associated types. A link is a tuple with one value for each end of the association, where each value is an instance of the type of the end...An association describes a set of tuples whose values refer to typed instances." [9, p.81]. The OMG UML Specification is somehow ambiguous in defining associations. An association is primarily considered to be a 'connection', but, in certain cases (whenever it has 'class-like properties'), an association may be a class: An association class is "[a] model element that has both association and class properties. An AssociationClass can be seen as an association that also has class properties, or as a class that also has association properties. It not only connects a set of classifiers but also defines a set of features that belong to the relationship itself and not to any of the classifiers." [9, p.118].

\subsection{Representing Formal and Material Relations}

An association $A$ between the classes $C_{1}, \ldots, C_{n}$ of a conceptual model can, in principle, be understood in our framework as a relation $R$ between the corresponding universals $\mathrm{U}_{1}, \ldots, \mathrm{U}_{\mathrm{n}}$ whose extension consists of all tuples corresponding to the links of A. However, current conceptual modeling languages (including UML) do not distinguish between formal and material relations. In figure 2, an example of a formal relation is the relation of temporal precedence between Symptoms. In this model, the unstereotyped classes (Person, Patient and Medical Unit) represent object universals; the quality universal Symptom is represented by a class with the corresponding stereotype; Finally, the intrinsic property start date of a symptom (a universal whose instances are qualities of a quality) is not represented directly but instead by its associated quality structure, the tridimensional DateDomain. The representation rules used in this model amount to the modeling profile proposed in [1,2] and are discussed in depth there.

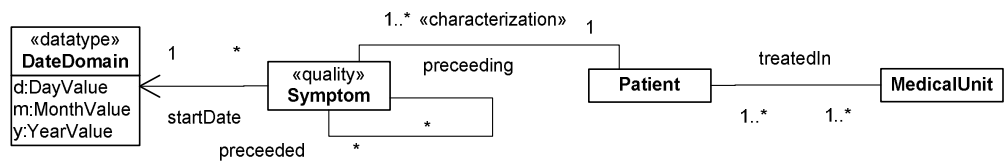

Figure 2. Representing Objects, Qualities, Quality Structures and Relations.

It is easy to see that the relation of Precedence in figure 2 is a domain formal relation since it is completely reducible to intrinsic qualities of the involved relata. It is common in conceptual modeling and knowledge representation languages that a number of formal meta-properties (e.g., reflexivity, symmetry, transitivity) are defined for relationships (e.g., OWL, F-LOGIC). In the specific case of precedence, these metaproperties are irreflexivity, anti-symmetry and transitivity and, hence, precedence is a strict partial ordering relation between symptoms that depends only on the starting date of each of them. Can we provide an explanation for these meta-properties? 
As we have discussed in section 2, the immediate relata of domain formal relations are not objects but qualities. Take, for example, the relations of taller than, heavier than and precedence. All these relations can be reduced to relations between qualities: $\mathrm{x}$ is taller than $\mathrm{y}$ iff height $(\mathrm{x})>\operatorname{height}(\mathrm{y}) ; \mathrm{x}$ is heavier than $\mathrm{y}$ iff weight( $\mathrm{x})>$ weight $(\mathrm{y})$; $\mathrm{x}$ preceeds $\mathrm{y}$ iff $\operatorname{startDate}(\mathrm{x})<\operatorname{startDate}(\mathrm{y})$, in which height, weight and startDate are attribute functions mapping the objects $\mathrm{x}$ and $\mathrm{y}$ to the corresponding qualia. All three quality structures involved in these expressions have a linear structure ordered by the < relation. By making this analysis explicit, it becomes evident that precedence is an ordered relation because the qualities founding this relation are associated with a ordered quality structure. In general, we can state that the metaproperties of a formal relation $\mathrm{R}_{\mathrm{F}}$ can be derived from the meta-properties of the relations between qualia associated with the qualities founding this relation $R_{F}$.

Now, take for instance the relation treatedIn between Patient and Medical Unit in figure 2. This relation requires the existence of a third entity, namely an individual Treatment mediating a particular Patient and a particular Medical Unit in order for the relation to hold, i.e., it is an example of a material relation. There is a specific practical problem concerning the representation of material relations as standard associations as depicted in figure 2. This problem, mentioned in [10], is caused by the fact that the standard notation for associations collapses two different types of multiplicity constraints. In this particular example, the model represents that each Patient can be treated in one-to-many Medical Units and that each medical unit can treat one-tomany patients. However, this statement is ambiguous since many different interpretations can be given to it, including the following: (i) a patient is related to only one treatment in which possibly several medical units participate; (ii) a patient can be related to several treatments to which only one single medical unit participates; (iii) a patient can be related to several treatments to which possibly several medical units participate; (iv) several patients can be related to a treatment to which several medical units participate, and a single patient can be related to several treatments. The cardinality constraint that indicates how many patients (or medical units) can be related to one instance of Treatment is named single-tuple cardinality constraint. Multiple-tuple cardinality constraints restrict the number of treatments a patient (or medical unit) can be related to.

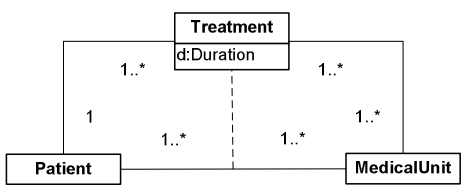

Figure 3. Explicit representation of single-tuple and multiple-tuple cardinality constraints.

How shall we represent a material relation in a conceptual modeling language such as UML such that the aforementioned problem could be addressed? Let us follow for now this (tentative) principle: a material relation $\mathrm{R}_{\mathrm{M}}$ of the domain may be represented in a conceptual model by representing the relator universal associated with the relation as an association class. By applying this principle to the treatedIn relation aforementioned we obtain the model of figure 3 . In this model, by modeling the relator universal Treatment as an association class one can explicitly represent both types of cardinality constraints. 
The reader should notice that the aforementioned problem is not at all specific to this case. For another example of a situation where this problem arises see figure 4.a. In this case, the (material) relation statement is that: (a) a customer purchases one-tomany purchase items from one-to-many suppliers; (b) a supplier supplies one-tomany purchase items to one-to-many customers; (c) a purchase item can be bought by one-to-many customers from one-to-many suppliers. PurchaseFrom is a material relation induced by the relator universal Purchase, whose instances are individual purchases. Therefore, we have that $\left[\mathbf{a}_{1}, \mathbf{a}_{2}, \mathbf{a}_{3}\right]:: \mathbf{R}_{\text {purchFrom}}($ Customer, PurchaseItem, Supplier) $\leftrightarrow a_{1}::$ Customer $\wedge a_{2}::$ PurchaseItem $\wedge a_{3}::$ Supplier $\wedge \exists p$ (p::Purchase $\left.\wedge m\left(\mathbf{p}, \mathrm{a}_{1}\right) \wedge \boldsymbol{m}\left(\mathrm{p}, \mathrm{a}_{2}\right) \wedge \boldsymbol{m}\left(\mathrm{p}, \mathrm{a}_{3}\right)\right)$. In other words, for this relation to hold between a particular Customer, a particular PurchaseItem, and a particular Supplier, they must be mediated by the same Purchase instance.
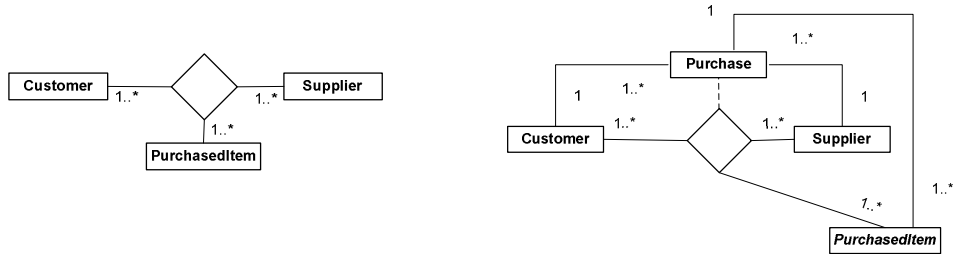

Figure 4. Example of a material relation with ambiguous (a-left) and (b) with explicit representation of cardinality constraints.

Once more, we can see that the specification in figure 4.a collapses single-tuple and multiple-tuple cardinality constraints. For this reason, thereare several possible ways of interpreting this model, including the following: (i) In a given purchase, a Customer participates by buying many items from many Suppliers and a customer can participate in several purchases; (ii) In a given purchase, many Customers participate by buying many items from many Suppliers, and a customer can participate in only one purchase; (iii) In given purchase, a Customer participates by buying many items from a Supplier, and a customer can participate in several purchases; (iv) In given purchase, many Customers participate by buying many items from a Supplier, and a customer can participate in several purchases. By depicting the Purchase universal explicitly (such as in figure 4.b), we can make explicit the intended interpretation of the material PurchaseFrom relation, namely, that in a given purchase, a Customer buys many items from a Supplier. Both customer and supplier can participate in several purchases. Although a purchase can include several items, each item in this model is a unique exemplar and, hence, can only be sold once.

Now it is important to emphasize that this problem is specific to material relations. Formal relations are represented by sets of tuples, i.e., an instance of the relation is itself a tuple with predefined arity. In formal relations, cardinality constraints are always unambiguously interpreted as being multiple-tuple, since there is no point in specifying single-tuple cardinality constraints for a relation with predefined arity. Hence, formal relations can be suitably represented as standard UML associations. One should notice that the relations between Patient and Treatment, and Medical Unit and Treatment are formal relations between universals (mediation). This is important to block the infinite regress that arises if material relations were required to relate 
these entities. The same holds for the pairwise associations between Customer, Supplier and PurchaseItem, on one hand, and Purchase on the other.

\subsection{An alternative to Association Classes}

At first sight, it seems to be satisfactory to represent a material relation by using an association class to model a relator universal that induces this relation. Nonetheless, the interpretation of this construct in UML is quite ambiguous w.r.t. defining what exactly counts as instances of an association class. We claim that the association class construct in UML exemplifies a case of construct overload in the technical sense discussed in [11]. This is to say that there are two distinct ontological concepts that are represented by this construct.

To support this claim, we make use of the following (overloaded) semantic definition of the term as proposed by the pUML (precise UML) community: "an associaton class can have as instances either (a) a n-tuple of entities which classifiers are endpoints of the association; (b) a n+1-tuple containing the entities which classifiers are endpoints of the association plus an instance of the objectified association itself" [12]. Take as an illustration the association depicted in figure 3 . In case (a), TreatedIn can be directly interpreted as a relation, whose instances are pairs $[a, b]$, whereas $a$ is patient and $b$ is medical unit. In this case, $[a, b]$ is an instance of TreatedIn iff there is a relator Treatment connecting $a$ and $b$. In interpretation (b), TreatedIn is what is named in [3] a Factual Universal. In short, if the relator $r$ connects (mediates) the entities $\mathrm{a}_{1}, \ldots, \mathrm{a}_{\mathrm{n}}$ then this yields a new individual that is denoted by $\left\langle\mathrm{r}: \mathrm{a}_{1}, \ldots, \mathrm{a}_{\mathrm{n}}\right\rangle$. Individuals of this latter sort are called material facts. For every relator universal $\mathrm{R}$ there is a set of facts, denoted by $\operatorname{facts}(\mathrm{R})$, which is defined by the instances of $\mathrm{R}$ and their corresponding arguments. Therefore, an instance of TreatedIn in this case could be the material fact $\left\langle\mathrm{t}_{1}\right.$ : John, MedUnit $\left.{ }_{\# 1}\right\rangle$, whereas John is a Patient, MedUnit ${ }_{\# 1}$ is a Medical Unit and $t_{1}$ is a treatment relator.

As a trope, a relator can bear other tropes. For example, in figure 3 the temporal duration of a Treatment is a quality of the latter. Moreover, a relator can also be mediated by other relators such as, for instance, a relator universal Payment whose instances connect particular Treatments and Payers. For these reasons, between the two aforementioned interpretations for association classes, we claim that interpretation (b) should be favored, since it allows for the explicit representation of relators and their possible intrinsic and relational properties. However, there is still one problem with this representation in UML. Suppose that treatment $t_{1}$ mediates the individuals John, and the medical units MedUnit $\# 1$ and MedUnit ${ }_{\# 2}$. In this case, we have as instances of the association class Treatment both facts $\left\langle\mathrm{t}_{1}\right.$ : John, MedUnit $\left.{ }_{\# 1}\right\rangle$ and $\left\langle\mathrm{t}_{1}\right.$ : John, Me$\left.\mathrm{dUnit}_{\# 2}\right\rangle$. However, this cannot be represented in such a manner in UML. In UML, $\mathrm{t}_{1}$ is supposed to function as an object identifier for a unique tuple. Thus, if the fact $\left\langle\mathrm{t}_{1}\right.$ : John, MedUnit $\left.{ }_{\# 1}\right\rangle$ holds then $\left\langle\mathrm{t}_{1}\right.$ : John, MedUnit $\left.{ }_{\# 2}\right\rangle$ does not, or alternatively, John and MedUnit $_{\# 2}$ must be mediated by another relator. These are, nonetheless, unsatisfactory solutions, since it is the very same relator Treatment that connects one patient to a number of different medical units.

We therefore propose to represent relator universals explicitly as in figure 5. This model explicitly distinguishes the two entities: relator universals are represented by the stereotyped class «relator»; material relations are represented by a derived UML 
association stereotyped as «material». The dashed line between a material relation and a relator universal, represents that the former is derived from the latter (see derived from relation in section 2.5). To mark this difference to the similar graphic symbol in UML, we attach a black circle in the relator universal end of this relation. In this figure, a particular Treatment is existentially dependent on a single Patient and in a (immutable) group of medical units. This would mean in UML that for every association representing an existential dependency relation between a trope and the individual it depends on, the association end should have the frozen meta-attribute set to true on the side of the latter. This compound modeling construct should replace the ambiguous association class construct in UML. Unlike in figure 3, the entities representing a relator universal (the stereotyped class that replaces an association class), and the material relation (the association itself) are distinct entities. In fact, the latter is completely derived from the former (see definition 7). For instance, the relator universal Treatment and the material relation TreatedIn represent distinct entities and can possibly have different cardinalities, since the same relator $t_{1}$ can connect both the entities in [John, MedUnit $\left.{ }_{\# 1}\right]$ and [John, MedUnit $\left.{ }_{\# 2}\right]$. Nonetheless, the cardinality constraints of TreatedIn can be completely deduced from the existential dependency relations (mediation) between Treatment and the universals whose instances are the relata of TreatedIn, namely, Patient and MedicalUnit.

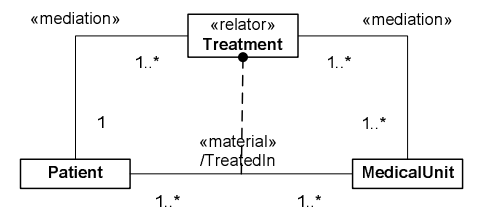

Figure 5. Model with explicit representation of a Relator Universal, a Material Relation, and a (formal) derivation relation between the two.

The benefits of this approach are even more evident in the case of n-ary relations with $\mathrm{n}>2$. Take the UML representation of a ternary relation in figure 4 . In this specification, we are forced to represent the minimum cardinality of zero for all association ends. As explained in the UML specification [9, p.82]: "For n-ary associations, the lower multiplicity of an end is typically 0 . If the lower multiplicity for an end of an nary association of 1 (or more) implies that one link (or more) must exist for every possible combination of values for the other ends". As recognized by the UML specification itself, n-ary associations in which there are tuples for every possible combination of the cross-product of the extension of the involved classes are atypical. Thus, in the majority of cases, the UML notation for n-ary associations completely looses the ability of representing real minimum cardinality constraints. Furthermore, as empirically demonstrated in [13], conceptual models without optional properties (minimum cardinality constraints of zero) lead to better performance in problem-solving tasks that require a deeper-level understanding of the represented domain.

The results of this section can be summarized in the following principle regarding the representation of formal and material relations in a conceptual model: In a conceptual model, any domain formal relation universal $\mathrm{R}_{\mathrm{F}}$ may be directly represented as a standard association whose links represent the tuples in the extension of $\mathrm{R}_{\mathrm{F}}$. Conversely, a material relation $\mathrm{R}_{\mathrm{M}}$ of the domain may be represented by a complex construct composed of: (i) a class stereotyped as «relator» representing the relator uni- 
versal. The relator universal is associated to classes representing mediated entities via associations stereotyped as «mediation»; (ii) a standard association stereotyped as «material» representing a material relation whose links represent the tuples in the extension of $R_{M}$; (iii) a dashed line with a black circle in one of the ends representing the formal relation of derivation between $\mathrm{R}_{\mathrm{M}}$ and the relator universal it derives from.

\section{A Critical Comparison to the BWW Approach}

The approach found in the literature that is closest to the one presented here is the socalled BWW approach presented in (e.g., $[5,11,14])$. In these articles, the authors report their results in mapping common constructs of conceptual modeling to an upper level ontology. Their approach is based on the BWW ontology, a framework created by Wand and Weber on the basis of the original metaphysical theory developed by Mario Bunge in [15].

In BWW, a property whose existence depends only on a single thing is called an intrinsic property. A property that depends on two or more things is called a mutual property. These concepts are analogous to our notions of intrinsic and relational trope universals. Nevertheless, in our approach properties are instantiated. Thus, our intrinsic properties can be better defined as universals whose instances inhere in a single individual, while relational properties are universals whose instances mediate multiple individuals. This marks an important distinction between the two approaches.

As demonstrated in [2], the ontological position behind the BWW approach (inherited from Bunge) is the substance-attribute view, whilst ours is a trope-theoretical one. Two consequences of their particular ontological choice are: (i) the denial of the existence of instances of properties; (ii) and the consequence denial of properties of properties (i.e., higher order properties). Thus, in BWW, only things (objects) possess properties. In particular, for the case of relational properties, this dictum leads to the following modeling principle: "Associations should not be modeled as classes" (rule 7 in [14]). This claim is not only perceived as counterintuitive by conceptual modeling practitioners (as shown by $[16,17]$ ), but, as discussed in depth [1,2], it is also controversial from a metaphysical point of view and puts BWW in a singular position among the foundational ontologies developed in the realm of computer science. Moreover, even if both ontological choices were deemed equivalent, there are a number pragmatic reasons for defending the acceptance of property instances and, hence, in favor of accepting also the representation of non-object universals as conceptual modeling types $[1,2]$. Examples include the proper representation of weak entities and structured datatypes [2] and, to cite an example demonstrated here, the explicit representation of relator universals (relational properties), which allows for the disambiguation of single-tuple and multiple-tuple cardinality constraints in associations.

To provide one more example of the importance of relators in conceptual modeling, suppose the situation in which one wants to model that students are enrolled in universities starting on a certain date. Following the proscription of mutual properties being modeled as entity types, Wand and colleagues propose an alternative model for this situation depicted in Figure 6.a [5].

We claim that it is rather counterintuitive to think about a model of this situation in these terms. According to [5], relationships representing mutual properties are equivalent to n-ary attribute predicates. In this case, what is startDate supposed to 
stand for? Is it a binary predicate that holds, for example, for John and UFES, like in startDate (John, UFES)? This seems to be an absurd conclusion. Thus, startDate should at least be a ternary predicate applied to, for instance, startDate (John, UFES, 14-2-2004). Now, suppose that there are many predicates like this one relating a student and a university. For example, the start-date of writing the thesis, the start-date of receiving a research grant, etc. We believe that, in this case, the authors would propose to differentiate the startDate depicted in figure 6.a by naming it startDateofEnrollment. But does not this move make it obvious that startDate is actually a property of the enrollment? In our approach, this can be explicitly modeled such as in figure 6.b. In contrast to figure 6.a, the model of 6.b makes an explicit distinction between a closed-linked relation between student and university and an indirect relation between student and start date.

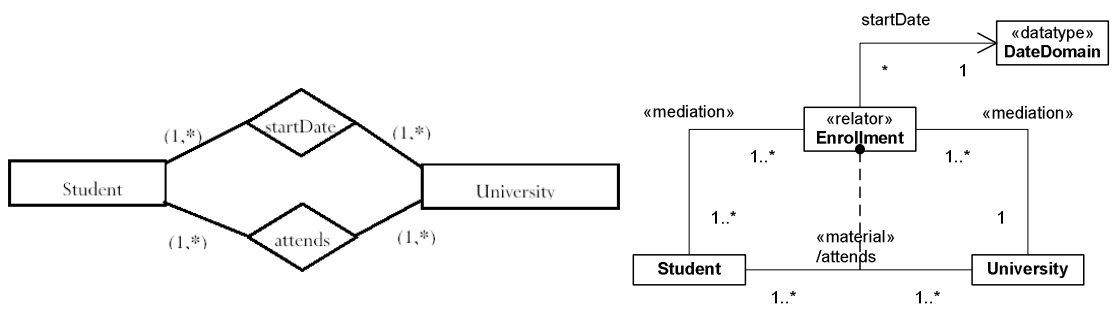

Figure 6. (a-left) An alternative modeling of "properties of properties" in the BWW approach (from [5]); (b) The representation of "properties of properties" in our approach.

\section{$5 \quad$ Final Considerations}

This article proposes an ontological theory of relations which makes a fundamental distinction between two different categories, namely, formal and material relations. This theory shows that only material relations stand for bonafide ontological relational properties (relational tropes or relators). Domain formal relations, in contrast, are simply useful logical constructions which do not stand for genuine properties of the things themselves but, instead, for the way we talk about these things. It is important to highlight that the theory presented here deals with domain relations as opposed to relations that form the meta-level structure of a modeling framework such as parthood, generalization/specialization, participation (in processes), existential dependence, among others. In our approach, these meta-relations have been formally treated elsewhere $[1,3]$.

Making the aforementioned distinction explicit is important from an ontological point of view since the very nature of these categories is uncovered. However, this also bears important consequences from a modeling perspective. Deciding whether an $\mathrm{n}$-ary term in the universe of discourse stands for a formal or material relation and, deciding which is the foundation of these relations, amounts to eliciting the very meaning of these terms. On one hand, uncovering the intrinsic tropes and associated quality structures underlying a domain formal relation can explain which are the formal meta-properties which should be described for that relation. On the other hand, recognizing and representing the relator universal underlying a material relation helps 
to disambiguate different sorts of cardinality constraints (a problem which is specific to material relations). Contrariwise, as demonstrated in [1], by not clearly representing relators and, due to the ambiguity of cardinality constraints, the standard notation for associations can collapse in a single representation, multiple relational properties with even contradictory semantics, which can be major source of interoperability problems. Furthermore, as discussed in depth in [18], the explicit representation of relator universals and their corresponding existential dependency relations provides a suitable mechanism for consistency preservation between static and dynamic conceptual models - an issue which we intend to give an ontological treatment in future works.

Acknowledgements This research has been partially supported by the funding agencies FAPES (INFRA-MODELA project) and FACITEC (MODELA project).

\section{References}

1. Guizzardi, G., Ontological Foundations for Structural Conceptual Models, PhD Thesis, University of Twente, The Netherlands, 2005.

2. Guizzardi, G.; Masolo, C.; Borgo, S. "In Defense of a Trope-Based Ontology for Conceptual Modeling: An Example with the Foundations of Attributes, Weak Entities and Datatypes, Proceedings of ER 2006, USA., Springer-Verlag, 2006.

3. Guizzardi, G.; Herre, H.; Wagner, G. "On General Ontological Foundations of Conceptual Modeling", , Proceedings of ER 2002, Finland., Springer-Verlag, 2002.

4. Batra, D.; Hoffler, J. A.; Bostrom, R. P. .Comparing representations with relational and EER models., Communications of the ACM, v.33 n.2, p.126-139, Feb. 1990.

5. Wand Y.,Storey V.C., Weber R. An ontological analysis of the relationship construct in conceptual modeling. ACM Trans. on Database Systems, 24(4):494-528, Dec., 1999.

6. Gärdenfors, P. 'Conceptual Spaces: the Geometry of Thought', MIT Press, USA, 2000.

7. Heller, B., Herre, H. Ontological Categories in GOL. Axiomathes 14: 71-90, 2004

8. Mulligan, K.; Smith, B. A Relational Theory of the Act., Topoi (5/2), p.115-30, 1986.

9. OMG, UML 2.0 Infrastructure Specification, Doc.\# ptc/03-09-15, Sep., 2003

10. Bock, C.; Odell, J. A More Complete Model of Relations and Their Implementation: Relations as Object Types., Journal of Object-Oriented Programming, Vol 10/3, Jun, 1997.

11. Weber, R. Ontological Foundations of Information Systems., Coopers \& Lybrand, Melbourne, Australia, 1997.

12. Breu, R. et al. 'Towards a Formalization of the Unified Modeling Language', Proceedings fo the 11th ECOOP, Jyväskylä, Finland, 1997.

13. Bodart, F., Patel, A., Sim, M., Weber, R. Should Optional Properties Be Used in Conceptual Modelling? A Theory and Three Empirical Tests, Information Systems Research, Vol.12, No. 4, December, pp.384-405, 2001.

14. Evermann J., Wand Y. Towards ontologically based semantics for UML constructs, Proceedings of ER 2001, Japan. Springer-Verlag, 2001.

15. Bunge M. Treatise on Basic Philosophy. Vol. 3. Ontology I. The Furniture of the World. D. Reidel Publishing, New York, 1997.

16. Veres, C.; Mansson, G. 'Cognition and Modeling: Foundations for Research and Practice', Journal of Information Technology Theory and Application, v.7, n.1, 93-10, 2005.

17. Hitchman, S. "An interpretive study of how practitioners use entity-relationship modeling in a ternary relationship situation", Comm. Assoc. for Inf. Systems, 11, 451-485, 2003.

18. Snoeck, M.; Dedede, G. .Existential Dependency: The Key to semantic integrity between structural and behavioral aspects of object types., IEEE Transactions on Software Engineering, Vol.24, No.4, Apr.1998. 